\title{
Investigation of visual acuity and residual refractive error after cataract surgery in patients with senile cataract by phacoemulsification
}

\author{
Saeed Rahmani ${ }^{1}$ Atefeh Farashi Beyramvand ${ }^{2}$, Seyyed Hadi Ali ${ }^{3}$, Alireza Akbarzadeh Baghban ${ }^{4}$, Haleh \\ Kangari $^{5}$ \\ 1. Lecturer, Department of Optometry, School of Rehabilitation, Shahid Beheshti University of Medical Sciences, Tehran, \\ Iran. ORCID ID:0000-0001-6330-4405 \\ 2. Master of Optometry, School of Rehabilitation, Shahid Beheshti University of Medical Sciences, Tehran, \\ Iran(Corresponding Author), Tel: 021-77548865, Email: atefeh.farashi1369@gmail.com, ORCID ID:0000-0002-2371-5565 \\ 3. Ophthalmologist, Shahid Rahimi Hospital, Khorramabad, Iran.ORCID ID: 0000-0002-8010-8243 \\ 4. Professor, Proteomics Research Center, Department of Biostatistics, School of Allied Medical Sciences, Shahid Beheshti \\ University of Medical Sciences, Tehran, Iran. ORCID ID: 0000-0002-0961-1874 \\ 5. Assistant Professor, Department of Optometry, School of Rehabilitation, Shahid Beheshti University of Medical Sciences, \\ Tehran, Iran. ORCID ID: 0000-0002-8298-9574
}

\begin{abstract}
Background and Aim: Vision improvement and reduction of refractive errors are considered as important goals of cataract surgery. The purpose of this study was to evaluate residual refractive errors after cataract surgery in the patients with senile cataract in Shahid Rahimi Hospital in Khorramabad City between 2017 and 2018.

Material and Methods: This cross-sectional study included 178 patients. Visual acuity, refractive errors and biometry of 192 eyes with senile cataract were evaluated before and one month after surgery. Refractive error and biometry measurements were performed with an autorefractometer and IOL master, respectively. SRK/T formula was used for IOL power calculation. We used paired T-test for analysis of data with normal distribution and nonparametric Wilcoxon test for the rest of data. $\mathrm{P}<0.05$ was considered statistically significant.

Results: The mean equivalent spherical refraction decreased from $-0.7 \pm 0.22$ before surgery to $-0.04 \pm 0.06$ after surgery $(\mathrm{P} \leq 0.001)$. Before and after surgery, the mean values for corrected visual acuity were $0.42 \pm 0.01 \mathrm{D}$ and $0.91 \pm 0.01 \mathrm{D}(\mathrm{P} \leq 0.0001)$ and the mean values for axial length of the eyes were $23.13 \pm 0.05$ and $23.07 \pm 0.05$, respectively $(\mathrm{P} \leq 0.001)$. The mean values for anterior chamber depth of the eyes was $3.07 \pm 0.02 \mathrm{~mm}$ before the surgery which changed to $3.73 \pm 0.03 \mathrm{~mm}$ after the surgery $(\mathrm{P} \leq 0.0001)$.
\end{abstract}

Conclusion: The results showed that refractive errors $(\% 86)$ after cataract surgery were within acceptable range $( \pm 1.00 \mathrm{D})$ and the best corrected visual acuity was better than 0.5

Key words: Visual acuity; Cataract surgery; Refractive error

Received: Sep 23, $2019 \quad$ Accepted: Jan 14, 2020

How to cite the article: SaeedRahmani, AtefehFarashiBeyramvand, SeyyedHadi Ali,
AlirezaAkbarzadehBaghban, HalehKangari. Investigation of Visual Acuity and Residual
Refractive Error after Cataract Surgery in Patients with Senile Cataract with Phacoemulsification.
SJKU. 2020; 25 (3): 23-30 Copyright $\bar{C} 20 \overline{1} \overline{8}$ the Author (s).Published by Kurdistan University of Medical Sciences. This is an open access article distributed under the terms of the Creative Commons Attribution-Non Commercial License 4.0 (CCBYNC), where it is permissible to download, share, remix, transform, and buildup the work provided it is properly cited. The work cannot be used commercially without permission from the journal 


\section{بررسى حدت بينايى و عيوب انكسارى باقيمانده پِ از عمل جراحى بيماران با آب مرواريد}

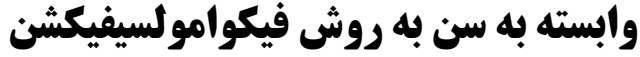

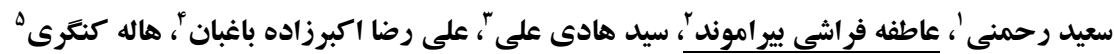

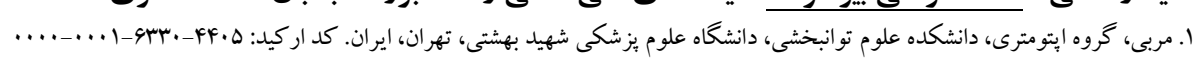

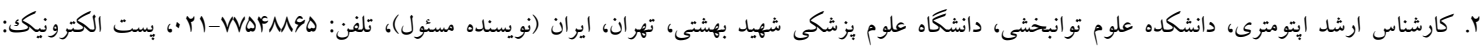

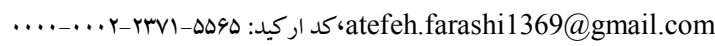

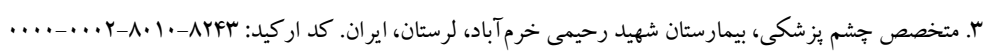

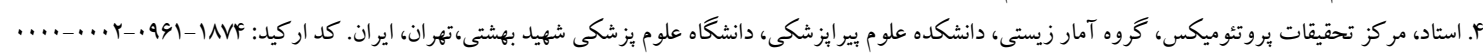

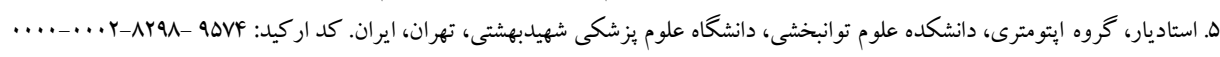
جكيله زمينه و هدف: بهبود ديد و كاهش عيوب انكسارى از اهداف مهم عمل جراحى آب مرواريد محسوب مىشود. هدف از اين

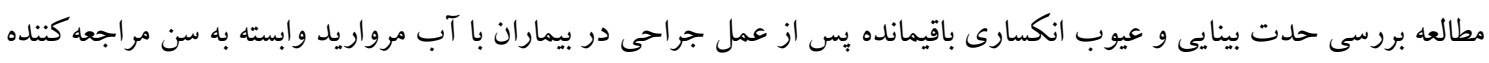

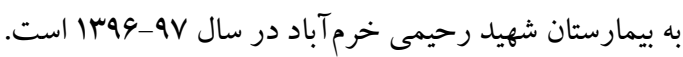
مواد و روشها: در اين مطالعه مقطعى علاوه بر عيوب انكسارى، حدت بينايى و بيومترى 19 إخشم (از IV9 بيمار) مبتلا به آب مرواريد وابسته به سن قبل و يكك ماه بعد ازعمل جراحى بررسى شد. اندازهيرى عيب انكسارى با اتورفر كتومتر، بيومترى توسط دستگاه Master و محاسبه قدرت لنز داخل جشمى با فرمول SRK/T انجام گرفت. جهت انجام تحليل آمارى در صورت توزيع نرمال دادهها از آزمون تى تست زوجى و در غير اين صورت از تست نايارامترى ويلكاكسون استفاده شد و سطح معنىدارى آزمون كمتر از هـ • • در نظر كرفته شد.

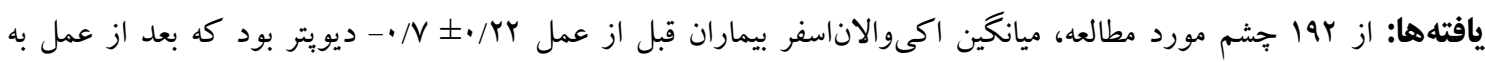

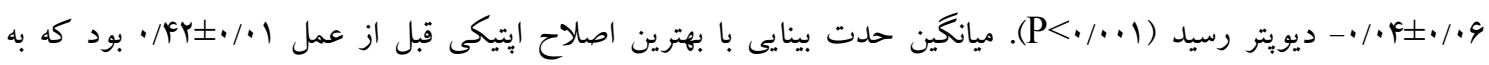

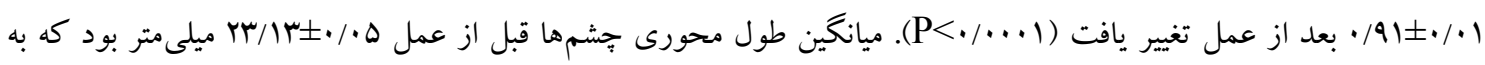

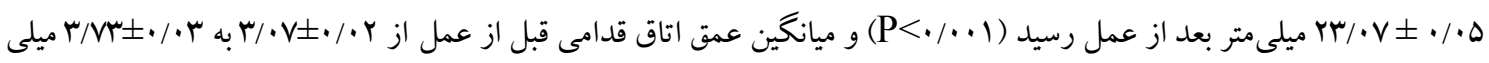

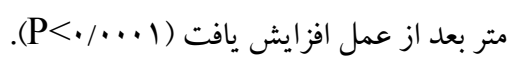

نتيجه كيرى: يافتهها نشان مىدهد كه عيوب انكسارى در بيش از وه1 درصد از بيماران مورد مطالعه بعد از عمل در محدوده قابل

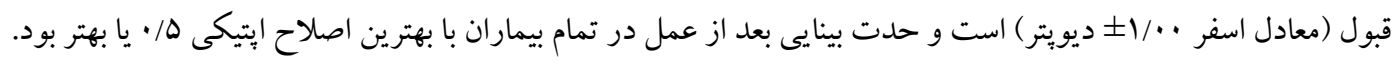

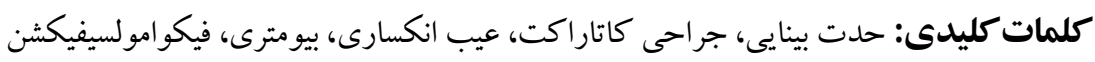
وصول مقاله : 19M/V/1 اصلاحيه نهايى: 
حدت بينايى اصلاح شده ضعيف قبل از عمل بهعنوان عوامل

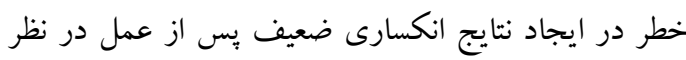
كرفته مىشوند(1)). لذا با توجه به اهميت مقدار عيوب

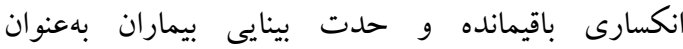

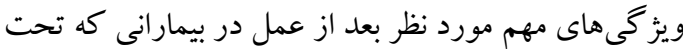

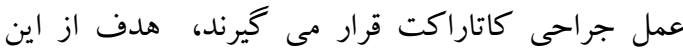
مطالعه بررسى عيوب انكسارى باقيمانده، حدت بينايى و تغييرات بيومترى بِ از عمل جراحى آب مرواريد وابسته به به بهاب سن در بيماران مراجعه كنده به بيمارستان شهيد رحيمى خرم آباد درسال 9V-9 دوسا است.

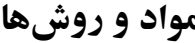

در اين مطالعه مقطعى، تعداد 19 جشم از إ IV9 بيمار مبتلا به كاتاراكت وابسته به سن قبل و بعد از عمل جراحى لئ كاتاركت مورد ارزيابى قرار گرفتند. اين مطالعه در سال 

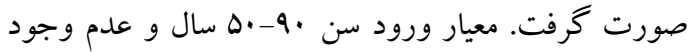

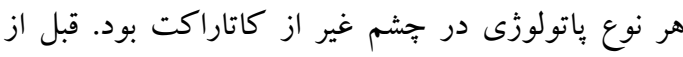
عمل، جهت تعيين عيب انكسارى از اتورفركتومتر استفاده شد و حدت (Topcon RM 8800, Japan) بينايى بدون اصلاح إيتيكى و با اصلاح إيتيكى به وسيله

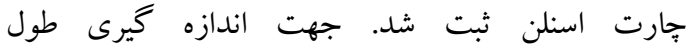
محورى،كراتومترى و محاسبه قدرت لنز داخل جشمى از IOL Master (Zeiss 500, Germany) دستگاه استفاده شد. جهت محاسبه قدرت لنز داخل جشمى از فرمول SRK/T يز شك مورد جراحى به روش فيكو امولسيفيكيشن و كاشت لنز داخل جشمى قرار گرفتند. بررسى ها شامل معاينه قبل از

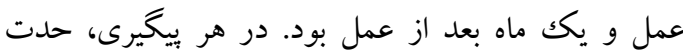

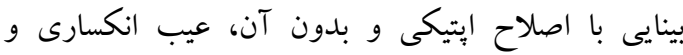

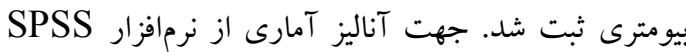

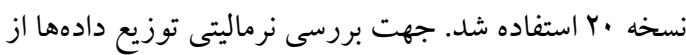

مقدمه كاتاراكت يا آب مرواريد به كدورت عدسى جشم گفته مى شود كه مانع از رسيدن نور به شبكيه جشم شده؛ بنابراين باعث كاهش ديد بيمار مىشود(r, 1). با اينكه انواع مختلفى از كاتاراكت وجود دارد؛ ولى مهمترين علت ايجاد آن تغييراتى در عدسى جشم است كه در اثر افزايش سن اتفاق

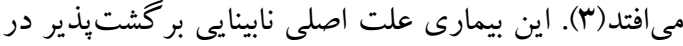
سر اسر جهان است(ه, \&). خارج نمودن عدسى كدر خشم از لاز طريق جراحى و كار كذارى لنز مصنوعى تنها راه اثبات شده درمان كاتاراكت است(^-9). عمل جراحى كاتاراكت روشى با موفقيت بالا و عوارض كمى است كه دوره بهبود يس از آن نيز كوتاه است. در اين عمل بيشينى عيب انكسارى پس از عمل بر روى عملكرد بينايى بيمار و در نتيجه رضايت وى تأثير بسز ايى دارد( •ا, 9). در يكك مطالعه فراوانى كاتاراكت در ايران حدود است(ه)؛ بنابراين نياز به عمل جراحى كاتاراكت بالا است. دستيابى به وضعيت انكسارى مورد نظر و به دنبال آن ديد بيمار بعد از عمل جراحى بسيار مهم است(rا, 11)؛ در نتيجه عمل جراحى كاتاراكت روى كيفيت زندگى بيماران

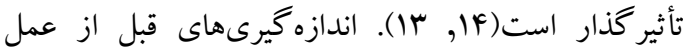
جراحى نقش مهمى در ميزان عيب انكسارى باقيمانده پِ از ازئ عمل دارد(Y)). اخرجه با وجود دستخاههاى اندازهخيرى و محاسبه دقيق لنزهاى داخل جشمى و همجنين روشهاى جراحى جديد و دقيق عمل جراحى آب مرواريد با موفقيت

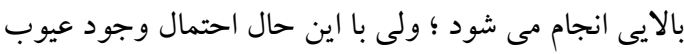

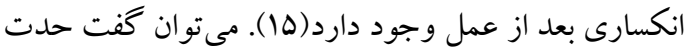
بينايى و عيب انكسارى از اجزاى مهم توانبخشى بينايى بعد

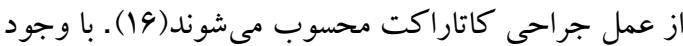
اينكه در اكثر موارد هدف از جراحى كاتاراكت ضمن درمان آن ايجاد امترويى(عدم وجود عيب انكسارى) بعد از ازتران عمل است؛ ولى در بسيارى از موارد اين نتيجه حاصل

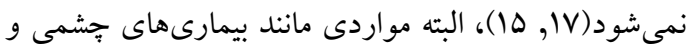




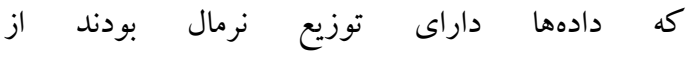

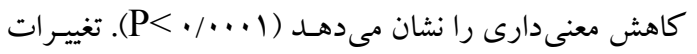

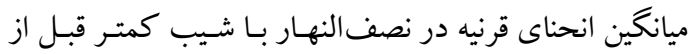

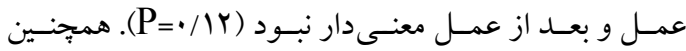
تغييرات قبل و بعد از عمل ميانگين انحناى قرنيه در نصف-

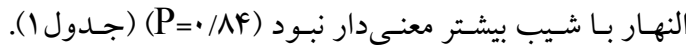

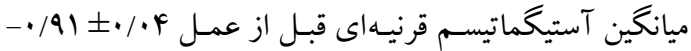

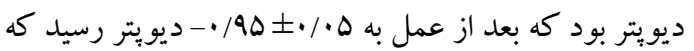

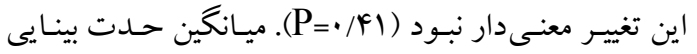

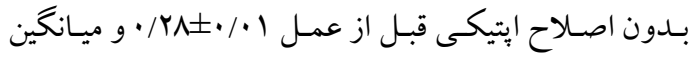

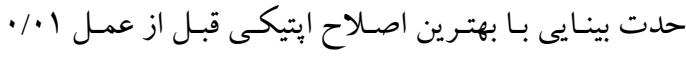

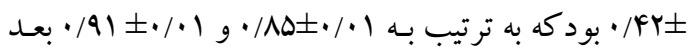

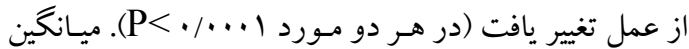

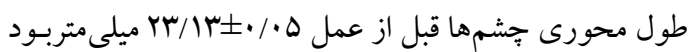

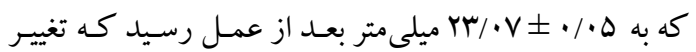

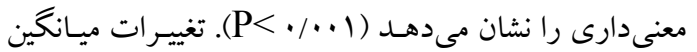

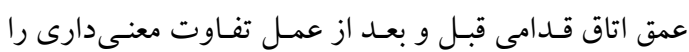

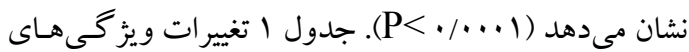

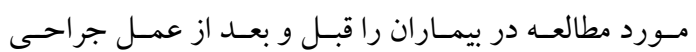

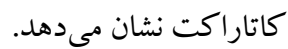

آزمون كولمو گروف-اسميرنوف استفاده شد، در مواردى آزمون تى تست زوجى و در مواردى كه توزيع دادهها نرمال

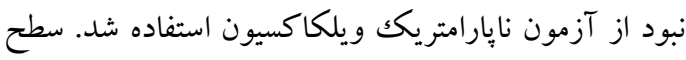
معنىدارى آزمونها هـ/P> در نظر كرفته شد. اين مطالعه در كميته اخلاق دانشگاه علوم بزشكى شهيد بهشتى با كد فد IR.SBMU.RETECH.REC.1396.1398

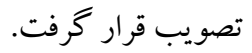

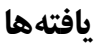

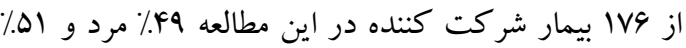

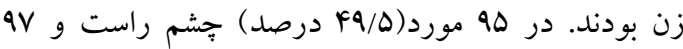

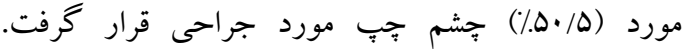
ميانگين سنى بيماران ميانكين عيب انكسارى اسفريكك بيماران قبل از عمل بيمان

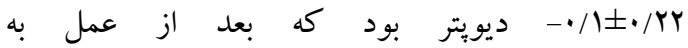

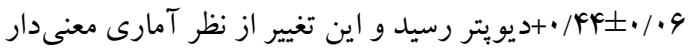

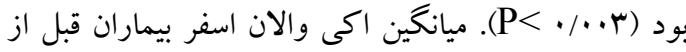

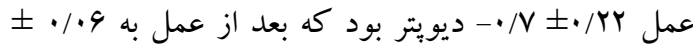

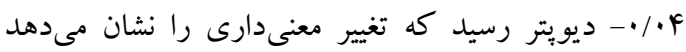
$(\mathrm{P}<\cdot / \cdots 1)$

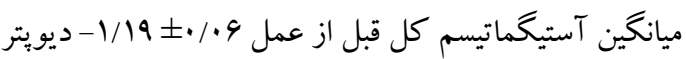

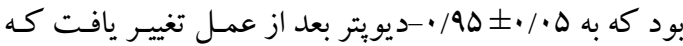
جدول 1. ويزكى هاى جشمى مورد بررسى در بيماران مورد مطالعه، قبل و بعد از عمل جراحى كاتاراكت

\begin{tabular}{|c|c|c|c|}
\hline \multirow[t]{2}{*}{ معنى دارى تغييرات } & بعد از عمل (ميانگين و & قبل از عمل (ميانگين و & ويخُكى \\
\hline & انحراف معيار) & انحراف معيار) & \\
\hline \multirow{2}{*}{$\mathrm{P}<\cdot / \cdot r$} & $+\cdot / 4 \varphi \pm \cdot / \cdot q$ & $-\cdot / / \pm \cdot / r Y$ & عيب انكسارى اسفريك \\
\hline & & & (به ديويتر) \\
\hline $\mathrm{P}<\cdot / \cdot \cdot \mathrm{l}$ & $-. / . \varphi \pm \cdot / \cdot 4$ & $-\cdot / V \pm \cdot / r Y$ & اكى والان اسفر (به ديويتر) \\
\hline $\mathrm{P}<\cdot / \cdots \cdot$ & $-\cdot / 9 \Delta \pm \cdot / \cdot \Delta$ & $-1 / 19 \pm \cdot / \cdot 9$ & آستيخماتيسم كل (به ديويتر) \\
\hline \multirow[t]{2}{*}{$\mathrm{P}=\cdot / \mathrm{Tr}$} & $F F / Y I \pm \cdot / Y r$ & $k F / r q \pm \cdot / l 1$ & انحناى قرنيه در نصف النهار با \\
\hline & & & شيب كمتر (به ديويتر) \\
\hline \multirow[t]{2}{*}{$\mathrm{P}=\cdot / \wedge f$} & $r \Delta / / V \pm \cdot / l Y$ & $r \Delta / \backslash \Lambda \pm \cdot / \Lambda Y$ & انحناى قرنيه در نصف النهار با \\
\hline & & & شيب بيشتر (به ديويتر) \\
\hline
\end{tabular}




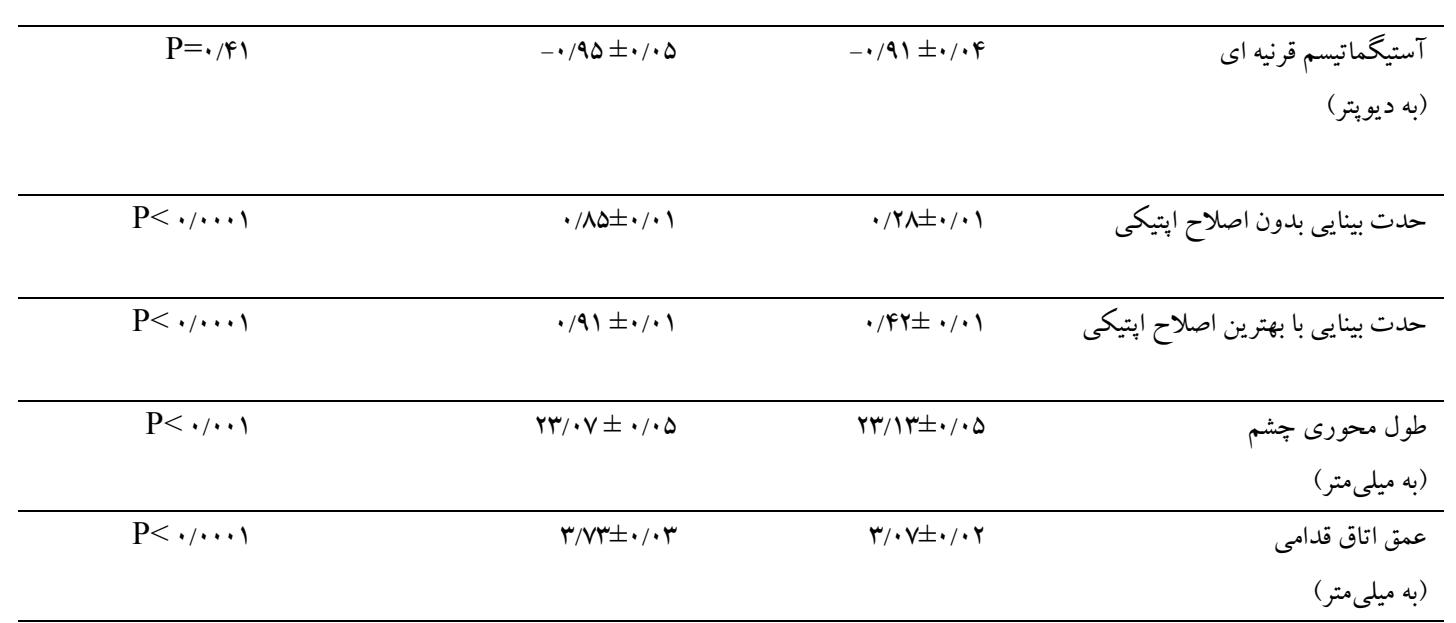

است؛ ولى اين تغييرات از نظر كلينيكى قابل ملاحظه نبوده و بحث اين تغيير را مىتوان به اين مسئله نسبت داد كه جون در اين مطالعه حدت بينايى و عيوب انكسارى باقيمانده و نيز كريستالين لنز داراى كاتاراكت حجيم بوده و زمانى كه با لنز

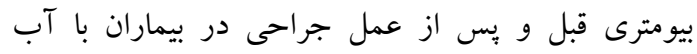

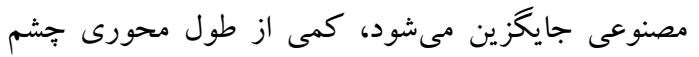

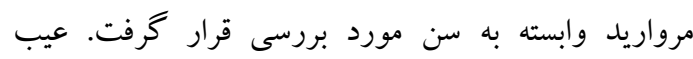

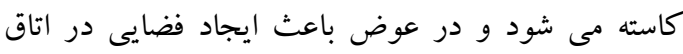

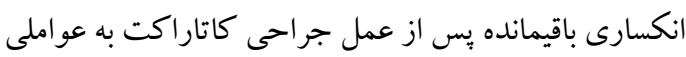
قدامى و افزايش در عمق آن شده است. از جمله روش بيومترى و فرمول مورد استفاده جهت تعيين

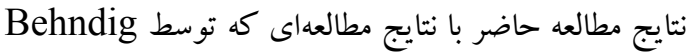
و همكاران (Y.MP) در سوئد انجام شده است قابل مقايسه

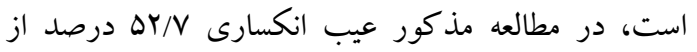

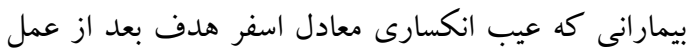
آنها در محدوده (ه/•tديويتر) بود پس از عمل در اين محدوده قرار داشتند. اطلاعات بيماران در اين مورد مطالعه از سيستم ثبت ملى كشور مذكور استفاده شده و جون در آن سيستم، اطلاعات عيوب انكسارى (اسفر و سيلندر) قبل از عمل، طول اتاق قدامى و فرمول مورد استفاده جهت تعيين قدرت عدسى داخلى جشم ثبت نشده است، در اين موارد امكان مقايسه نتايج بعد از عمل با مقادير قبل از عمل وجود لمدان

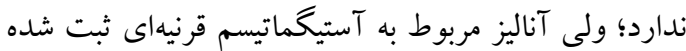
قبل و بعد از عمل در مطالعه فوق تغيير معنى ندارى نداشته(IV) كه با نتايج مطالعه حاضر همخوانى دارد.

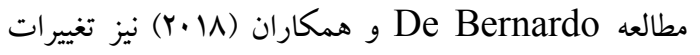
بدون معنىدارى قدرت قرنيهاى را قبل و يس از عمل

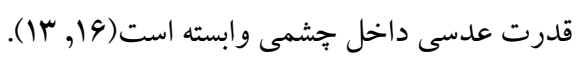
در مطالعه حاضر مقدار معادل اسفر درابا مورد (T) درصد) پس از عمل جراحى در محدوده مطلوب عيب انكسارى باقيمانده (ه/•t ديويتر) قرار داشت و در 199 مورد (ه/ه/ه درصد) پِ إز عمل جراحى در محدوده عيب

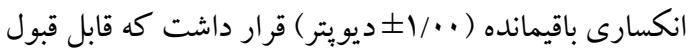
بوده، بهطورى كه مىتوان كَت كه بيماران با وجود مقادير

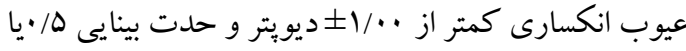

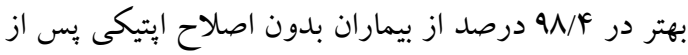

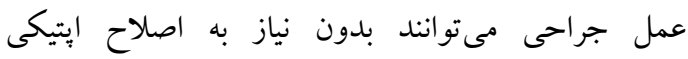
فعاليتهاى روزمره را به راحتى انجام دهند. اين مسئله نشان-

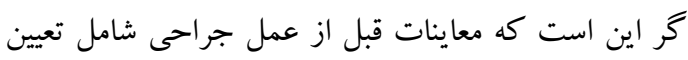
نمره لنز داخل جشمى و جراحى كاتاراكت به خوبى انجام شده است. نتايج بيومترى يس از عمل نشان داد كه هر جند كه طول محورى جشم ها پِ از عمل جراحى كمى كاهش يافته 
عيب انكسارى باقيمانده در محدودهى ه ••+ديويترو درحدود VF درصد از موارد در محدودهى ·_/ ديويتر بود(Y) كه با مطالعه حاضر تفاوت دارد و اختلاف بين نتايج دو مطالعه را مىتوان به دلايل زير مربوط دانست: در مطالعه فوق دادههاى ثبت شده در بروندههاى بيماران و سبس فراخوان بيماران انتخابى مورد بررسى قرار گرفته بود وبه مقدار عيب انكسارى قبل از عمل بيماران دسترسى وجود نداشت. بيومترى عمدتاً به روش اولتراسوند انجام شده و توسط افراد مختلف كم تجربه و با تجربه صورت كرفته بود. فرمول مورد استفاده در مطالعه فوق SRK-II بوده كه امروزه استفاده از اين فرمول توصيه نمىشود(Y), YY). در حالى كه در مطالعه حاضر حدت بينايى بعد از عمل در \$ ه/ درصد از بيماران بدون اصلاح ايتيكى و در ..1 درصد از

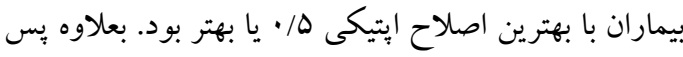

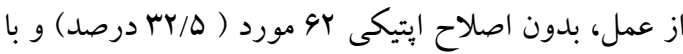
اصلاح إيتيكى F.ا مورد (D/D/D درصد) از بيماران حدت

$$
\text { بينايى • • • 1 كسب كرده بودند. }
$$

در مطالعهاى كه Murphy و همكاران (Y...Y) جهت بررسى عيب انكسارى و حدت بينايى بعد از عمل كاتاراكت، در بيش از VY درصد از موارد عيب انكسارى بعد از عمل بيماران در محدوده · /1 ديويتر بود، بعلاوه ه/4 درصد از بيماران فاقد مشكل جشمى ديكر با بهترين اصلاح إيتيكى به حدت بينايى ه/ · يا بهتر دست يافتند(19) كه با نتايج مطالعه حاضر مشابه بود. در مطالعه فوق جهت تعيين عدسى داخل جشمى با توجه به طولهاى متفاوت

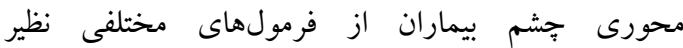
HofferQ و و جهت SRK/T قرنيه از دستگاه كراتومتر استفاده شده بود و روش بيومترى از نوع اولتراسوند بود. در مطالعه حاضر فقط از فرمول SRK/T دستگاه IOLMaster انجام شد. با توجه به اينكه در مطالعات بيشين به استفاده از فرمول SRK/T جهت اندازه گيرى قدرت عدسى داخل جشمى در عمل كاتاراكت
كاتاراكت به روش فيكوامولسيفيكيشن تائيدمى كند(19)؛ زيرا جراحى كاتاراكت با اين روش با ايجاد برشى كوجنى در قرنيه بيمار انجام مىشود؛ بنابراين تأثير جندانى روى وضعيت كر اتو مترى و قدرت قرنيه نخو اهد داشت. در مطالعه مشابهى كه توسط Kansal و همكاران (Y (Y) در كانادا به انجام رسيد( •Y) عيب انكسارى معادل اسفر پيس از عمل V/ دورصد از بيماران در محدوده مورد نظر (ه/• ديويتر) بود كه از فراوانى بيماران با عيب انكسارى معادل اسفر يس از عمل در مطالعه حاضر بالاتر است. اين در حالى است كه دستگاه مورد استفاده جهت تعيين نمره عدسى داخل جشمى در هر دو مطالعه فوق IOL Master بوده است كه اين تفاوت را مىتوان به فرمول استفاده شده جهت تعيين نمره عدسى داخل جشمى مربوط دانست كه در مطالعه حاضر SRK/T و در مطالعه مذكور Holladay1 بوده است. در مطالعه ديخرى كه توسط Karabela و همكاران جهت بررسى مقدار عيوب انكسارى باقيمانده بس (Y.IV) از عمل جراحى كاتاراكت انجام دادند، مشخص شد كه در 9Y/VD

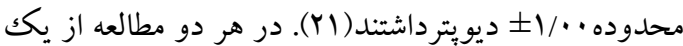
روش جهت جراحى و يكك فرمول (SRK/T) جهت تعيين قدرت عدسى داخل جشمى استفاده شد. در مطالعه حاضره/\$1 درصد از بيماران عيوب انكسارى بعد از عمل در محدوده فوق قرار داشت كه اين تفاوت مىتواند ناشى از روش بيومترى (اولتراسونوخرافى) مورد استفاده در مطالعه باشد. با اينكه جهت تعيين نمره عدسى داخل جشمى استفاده از هر دو روش امكانيذير است؛ ولى غير از مواردى خاص، روش بيومترى إيتيكى مورد استفاده در مطالعه حاضر

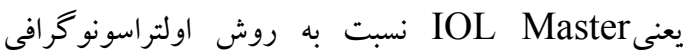
ارجحيت دارد و مى توان آن را بهعنوان استاندارد طلايى در بين روشهاى مورد استفاده در نظر گرفت(Y (Y), در مطالعهاى كه در ايران توسط هاشمى و همكاران ( به انجام رسيد، مشاهده شد كه در حدود 4\& درصداز موارد، 


$$
\begin{aligned}
& \text { محدودهى قابلقبول (اكى والان اسفر .1/ ديويتر) و } \\
& \text { در جشمهاى با طول محورى متوسط (YY/D } \\
& \text { حدت بينايى بعد از عمل در تمام بيماران با بهترين اصلاح } \\
& \text { ميلىمتر)(Y9) و جشمهاى با طول محورى متوسط تا بلند }
\end{aligned}
$$

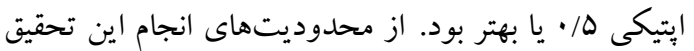

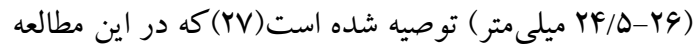

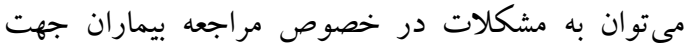

$$
\begin{aligned}
& \text { نيز طول محورى جشمهاى حدود \&ه درصد از بيماران در }
\end{aligned}
$$

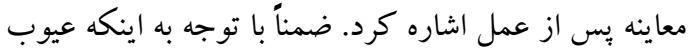

$$
\begin{aligned}
& \text { محدودههاى مذكور بوده كه اين مىتواند يكى از عوامل }
\end{aligned}
$$

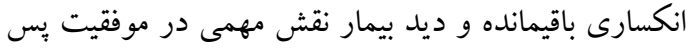

$$
\begin{aligned}
& \text { مؤثر در كاهش مقدار عيوب انكسارى باقيمانده بِ از عمل }
\end{aligned}
$$

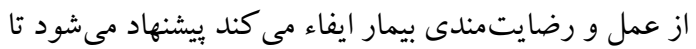

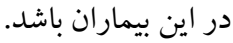

$$
\begin{aligned}
& \text { مطالعات مشابهى در ساير مراكز درمانى نيز انجام شود. } \\
& \text { خرم آباد تشكر و قدردانى مى گردد. }
\end{aligned}
$$
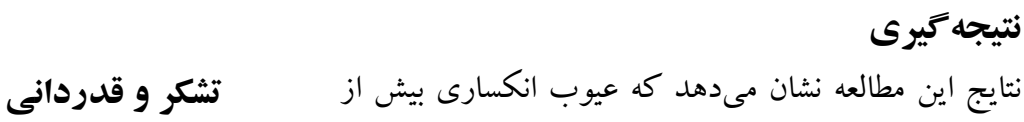

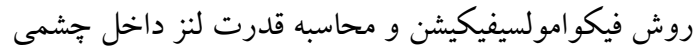

$$
\begin{aligned}
& \text { توسط دستگاه IOL Master با فرمول SRK/T در }
\end{aligned}
$$

1.Davis G. The Evolution of cataract surgery.Mo Med. 2016;113(1):58-62.

2.Das GK, Boriwal K, Chhabra P, Sahu PK, Kumar S. Presenile cataract and its risk factors: A case control study. J Family Med Prim Care. 2019;8(6):2120-23.

3.Aliancy JF, Mamalis N. Crystalline Lens and Cataract. The organization of the retina and visual system [Internet]. 2017;15:1-18. Available from: https://pubmed.ncbi.nlm.nih.gov/29356473/

4.Lee CM, Afshari NA. The global state of cataract blindness. Curr Opin Ophthalmol. 2017;28(1):98-103.

5.Shahdadi H, Aminifard MN, Balouchi A, Rafiemanesh H, Allahyari J. Frequency of cataract in Iran: A Meta-Analysis and Systematic Review. Middle East Afr J Ophthalmol. 2018;25(1):40-6.

6.Toh T, Morton J, Coxon J, Elder MJ. Medical treatment of cataract. Clin Exp Ophthalmol. 2007;35(7):664-71.

7.Friehmann A, Assia EI. New lenses in modern cataract surgery. Harefuah. 2019;158(2):126-9.

8.Crispim J, Chamon W. Lens: Management of cataract surgery, cataract prevention, and floppy iris syndrome. Handb Exp Pharmacol. 2017;242:163-78.

9.Elbaz U, Barkana Y, Gerber Y, Avni I, Zadok D. Comparison of different techniques of anterior chamber depth and keratometric measurements. Am J Ophthalmol. 2007;143(1):48-53.

10.Sales CS, Manche EE. Managing residual refractive error after cataract surgery. J Cataract Refract Surg. 2015;41(6):1289-99.

11. Melles RB, Kane JX, Olsen T, Chang WJ. Update on intraocular lens calculation formulas. Ophthalmology. 2019; 126(9):1334-35.

12.Fayette RM, Cakiner-Egilmez T. What factors affect intraocular lens power calculation?. Insight. 2015;40(4):15-8. 
... براسى هدت بينايى

13.Farmer L, Innes-Wong C, Bergman-Hart C, Casson RJ, Crompton J. Visual acuity, quality of life and visual function outcomes after cataract surgery in Bali. Ophthalmic Epidemiol. 2015;22(4):274-82.

14.Heemraz BS, Lee CN, Hysi PG, Jones CA, Hammond CJ, Mahroo OA. Changes in quality of life shortly after routine cataract surgery. Can J Ophthalmol. 2016;51(4):282-7.

15.Alio JL, Abdelghany AA, Fernandez-Buenaga R. Management of residual refractive error after cataract surgery. Curr Opin Ophthalmol. 2014;25(4):291-7.

16. Murphy C, Tuft SJ, Minassian DC. Refractive error and visual outcome after cataract extraction. J Cataract Refract Surg. 2002;28(1):62-6.

17.Behndig A, Montan P, Stenevi U, Kugelberg M, Zetterstrom C, Lundstrom M. Aiming for emmetropia after cataract surgery: Swedish National Cataract Register study. J Cataract Refract Surg. 2012;38(7):1181-6.

18.Lundström M, Dickman M, Henry Y, Manning S, Rosen P, Tassignon MJ, et al. Risk factors for refractive error after cataract surgery: Analysis of 282811 cataract extractions reported to the European Registry of Quality Outcomes for cataract and refractive surgery. J Cataract Refract Surg.2018;44(4):447-52.

19.De Bernardo M, Salerno G, Cornetta P, Rosa N. Axial length shortening after cataract surgery: New approach to solve the question. Translational vision science \& technology. 2018;7(6):34.

20.Kansal V, Schlenker M, Ahmed IIK. Interocular axial length and corneal Power Differences as Predictors of Postoperative Refractive Outcomes after Cataract surgery. Ophthalmology. 2018;125(7):972-81.

21.Karabela Y, Eliacik M, Kocabora MS, Erdur SK, Baybora H. Predicting the refractive outcome and accuracy of IOL power calculation after phacoemulsification using the SRK/T formula with ultrasound biometry in medium axial lengths. Clin Ophthalmol. 2017;11:1143-9.

22.Cech R, Utikal T, Juhaszova J. Comparison of optical and ultrasound biometry and assessment of using both methods in practice. Cesk Slov Oftalmol. 2014;70(1):3-9.

23.Hashemi H, Mohammadi F, Majdi M, Khabaz Khoob M, Zare Mehrjerdi H, Mazori A, et al. Residual refractive errors following cataract surgery and its determinants. Bina J Ophthalmol. 2010;15(4):263-74.

24.Cooke DL, Cooke TL. Comparison of 9 intraocular lens power calculation formulas. J Cataract Refract Surg. 2016;42(8):1157-64.

25.Cooke DL, Cooke TL. Prediction accuracy of preinstalled formulas on 2 optical biometers. J Cataract Refract Surg. 2016;42(3):358-62.

26.Lagrasta JM, Allemann N, Scapucin L, Moeller CT, Ohkawara LE, Melo LA, Jr, et al. Clinical results in phacoemulsification using the SRK/T formula. Arquivos brasileiros de oftalmologia. 2009;72(2):189-93.

27.Hoffer KJ. Clinical results using the Holladay 2 intraocular lens power formula. J Cataract Refract Surg. 2000;26(8):1233-7. 